\title{
Template Synthesis of Ordered Pt Nanorods in Porous Anodic Alumina
}

\author{
Yar-Ming Wang, Hong-Hsiang Kuo, and Mark W. Verbrugge \\ Materials and Processes Lab, GM R\&D Center, Warren, Michigan 48090 \\ Jie Lian and Lumin Wang \\ Department of Nuclear Engineering \& Radiological Sciences, University of Michigan, Ann Arbor, \\ Michigan 48109
}

Wei Zhou

School of Mechanical and Aerospace Engineering, Nanyang Technological University, Singapore 639798

High-purity aluminum was anodized to form an alumina coating layer (typically 10 to $30 \mu \mathrm{m}$ ) at the metal surface. The oxide coating layer has a duplex structure including a barrier layer and a porous layer having a hexagonal array of nano-pores. After anodizing, the metal is immersed in a bath containing a platinum electrolyte. An ac voltage is applied which deposits the metal salts in the base of the pores to form Pt nano-particles inside the pores by an electrolytic coloring process. The formed composite layer could be used as an oxidizing catalyst for automotive applications.

The morphologies of highly-ordered arrays of nano-pores in the amorphous alumina template (Fig. 1A) and the Pt metal nanorods (Fig. 1B) obtained after removing the front surface of the aluminum oxide have been observed using the electron beam in a FSEM/FIB dual beam system (FEI Nova 200 NanoLab). A uniform distribution in diameters has been observed for both ordered arrays of nanopores and Pt nanorods. The diameter of Pt nanorods $(12 \sim 20 \mathrm{~nm})$ is similar to that of amorphous nano-pores, suggesting the template formation of Pt nanorods. Cross-sectional TEM samples were prepared using FIB with a $\mathrm{Ga}^{+}$liquid metal ion source operated at $30 \mathrm{keV}$, and the interface microstructure has been characterized by scanning transmission electron microscope (STEM) using a JEOL 2010F microscope. Pt nanorods with the diameter of $\sim 20 \mathrm{~nm}$ were observed embedded along the nano-pores in the amorphous anodic alumina template. The average heights of Pt metal nanorods can be controlled approximately from $\sim 80 \mathrm{~nm}$ to $\sim 600 \mathrm{~nm}$ by varying the ac voltage and deposition time (Fig. 2). An amorphous alumina barrier layer $(\sim 20 \mathrm{~nm})$ has been found between the Pt nanorods and Al substrate. High-resolution TEM images from the interface (not shown here) clearly indicate that nano-pores do not entirely extend to the Al substrate, and Pt nanorods formed starting from the bottom of nano-pores, consistent with the template formation mechanism. Various areas showing diffraction contrasts have been found in the bright-field TEM images (not shown here). High resolution TEM image (Fig. 3A) shows crystalline feature in these areas. Amorphous regions also were observed in the Pt nanorods, and no modulation of chemical composition was detected between crystalline Pt nanoparticles and the amorphous regions. Pt nanorods were assembled by crystalline Pt nanoparticles and amorphous domains. The selected area diffraction pattern (Fig. 3A) of nanorods can be indexed by pure Pt with the fcc structure $(\boldsymbol{a}=0.39 \mathrm{~nm})$, suggesting the pure metallic feature of the nanorods, consistent with the energy-dispersive x-ray (EDS) measurement (Fig. 3C). These results clearly demonstrate the formation of pure metallic Pt nanorods in amorphous alumina matrix using highly-ordered arrays of nano-pores as templates. Pt nanorods were confined inside the nanopores, and assembled by crystalline Pt nanoparticles and some amorphous domains elongated along nano-pores. The Pt metal nanorods formed inside the nanopores of anodic alumina template may have a potential application as an oxidizing catalyst in the automotive industry. 

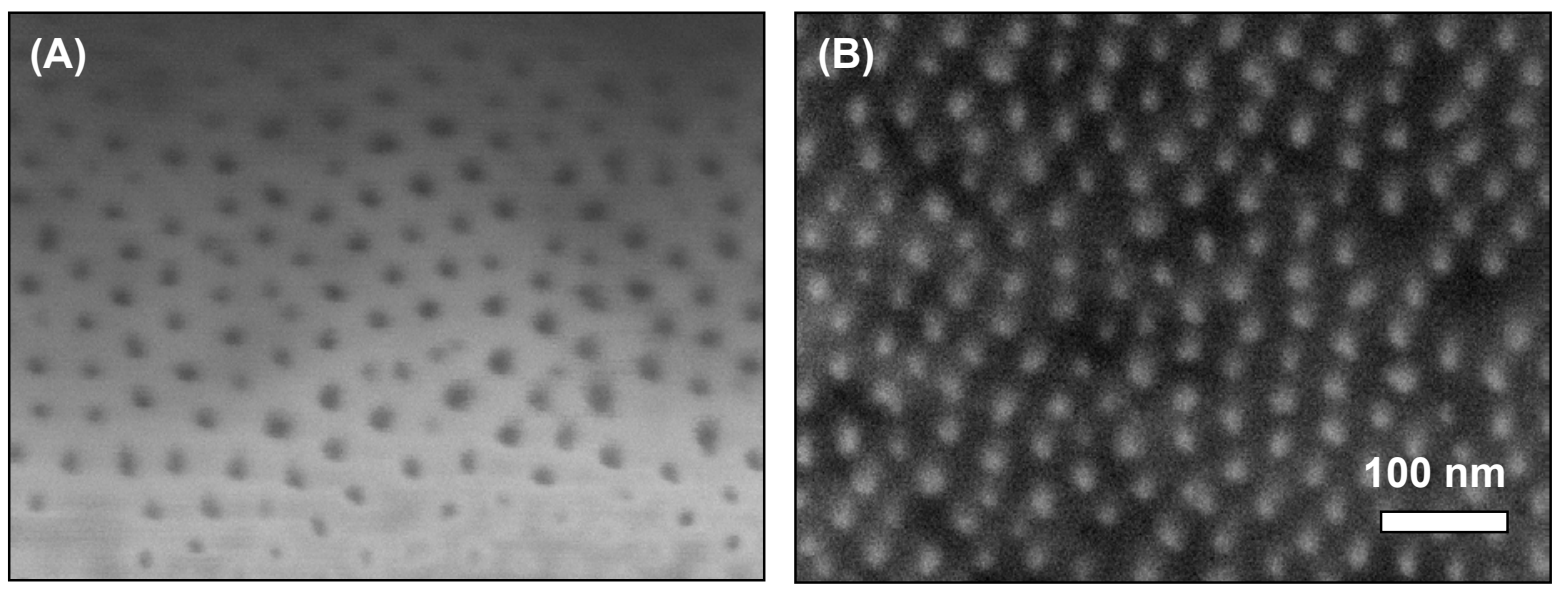

Fig. 1. SEM micrographs of highly-ordered hexagonal array of nano-pores in amorphous alumina template (A) and Pt nanorods (B).
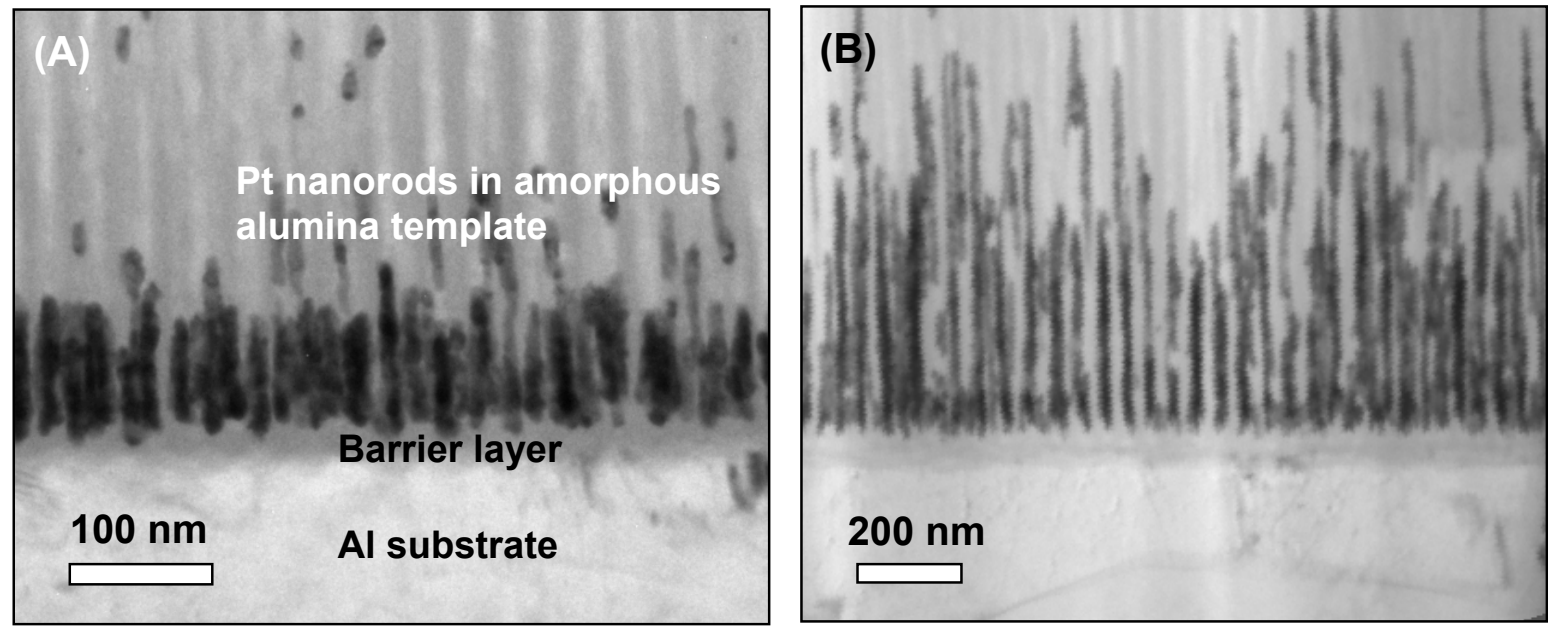

Fig. 2. Cross-sectional TEM images showing Pt nanorods in porous anodic alumina template by the electrochemical deposition (ac voltage: $8 \mathrm{~V}$ ) at different disposition times: (A) $30 \mathrm{~s}$ and (B) $600 \mathrm{~s}$. The average heigth of $\mathrm{Pt}$ metal nanorods can be controlled from $\sim 80 \mathrm{~nm}$ (A) to $\sim 600 \mathrm{~nm}(\mathrm{~B})$.
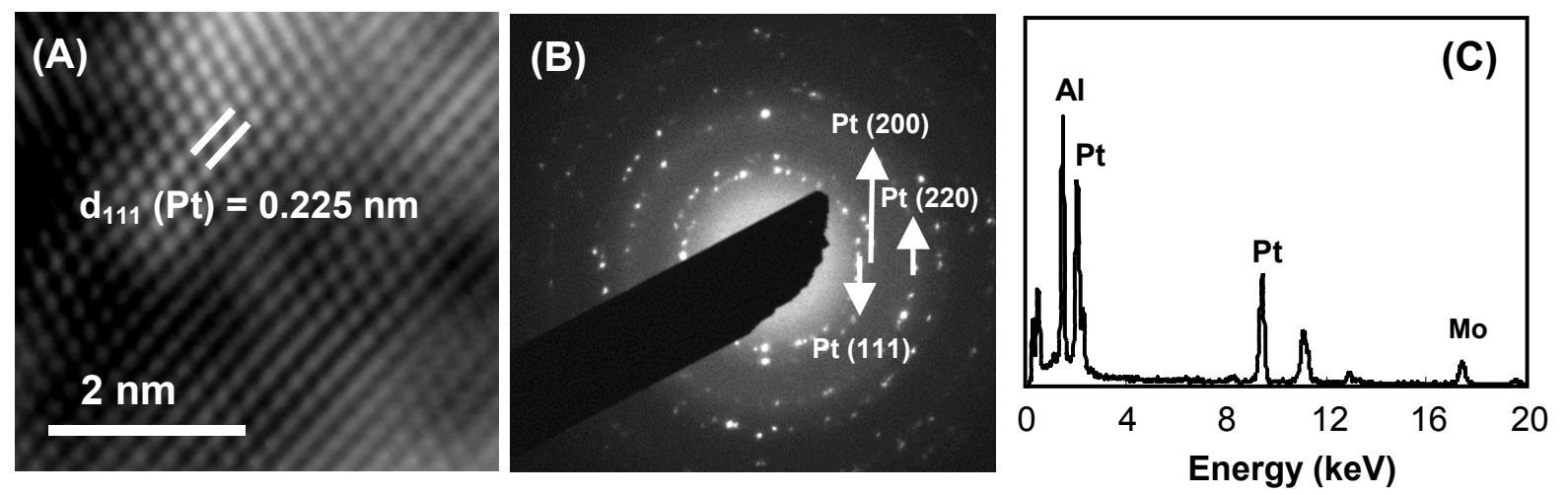

Fig. 3. High-resolution TEM image (A), selected-area diffraction (SAED) pattern (B) and the EDS spectrum (C) of Pt nanorods. 